\title{
Epidemiología descriptiva y genética molecular del cáncer de mama hereditario en Costa Rica
}

\author{
Laura García-Jiménez ${ }^{1,2}$, Gustavo Gutiérrez-Espeleta ${ }^{2} \&$ Steven A. Narod ${ }^{3}$ \\ 1. Facultad de Estudios Superiores Zaragoza (Diplomado en Investigación Clínica y Epidemiológica), Universidad \\ Nacional Autónoma de México, Distrito Federal, México; laura.garcia_j@ucr.ac.cr \\ 2. Escuela de Biología, Universidad de Costa Rica, San José, Costa Rica; gustavo.gutierrez@ucr.ac.cr \\ 3. Women's College Research Institute, Toronto, Ontario, Canada; steven.narod@wchospital.ca
}

Recibido 07-XI-2011. Corregido 20-IV-2012. Aceptado 29-V-2012.

\begin{abstract}
Descriptive epidemiology and molecular genetics of hereditary breast cancer in Costa Rica. Breast cancer is first in incidence and mortality among Costa Rican women. In 2011 there is a projected 1071 new cases. We selected 116 patients diagnosed with breast cancer and family history of breast cancer. A detailed family history and epidemiology data was obtained from each patient and a blood sample was processed for DNA extraction. We analyzed the prevalence of risk factors and BRCA1 and BRCA2 mutations. These mutations were detected by different techniques and were confirmed by direct sequencing. Six patients (5.2\%) had a mutation, five of which were in the BRCA2 gene and only one in BRCA1. We found that the average age of women who carry a mutation is lower than in non-carrier women. All other risk factors analyzed were not different among carrier and non-carrier women. In addition, these results and the increased incidence of breast cancer in recent years in Costa Rica, should promote an increase in prevention policies and the establishment of efficient detection methods that allow early disease diagnosis. Rev. Biol. Trop. 60 (4): 1663-1668. Epub 2012 December 01 .
\end{abstract}

Key words: breast cancer, hereditary, risk factors, Costa Rica.

El cáncer de mama $(\mathrm{CM})$ constituye un problema de salud pública a nivel mundial pues es la neoplasia más frecuente en la mujer. Comprende aproximadamente el $18 \%$ de todos los tipos de patologías femeninas y posee una de las mayores tasas de incidencia y mortalidad en el mundo (McPhearson et al. 2000).

Costa Rica ocupa el cuarto lugar en incidencia a nivel Latinoamericano. Según la Organización Panamericana de la Salud (2006), en relación con las tasas de incidencia y mortalidad de cáncer en las mujeres en Costa Rica, el cáncer de mama obtuvo el segundo lugar con una tasa de incidencia (ajustada por 100000 mujeres) de 38.52 en la población femenina. Para esta patología en particular, datos actuales muestran un incremento de más de un $45 \%$ en su incidencia para el período entre 1990-2001.
En relación con la tasa de mortalidad, en el 2005 ocupó el primer lugar con una tasa ajustada por 100000 mujeres de 10.8 (Ortiz 2007).

De acuerdo AICR (2008), entre las mujeres costarricenses esta neoplasia ocupa el primer lugar en incidencia y mortalidad, desplazando al cáncer gástrico. En el 2007 se diagnosticaron alrededor de 818 casos nuevos y para el 2011 se esperarían aproximadamente 1071 casos nuevos (Ministerio de Salud 2007).

El desarrollo de esta enfermedad se ha asociado con diversos factores de riesgo, tales como la historia familiar, edad, factores ginecoobstétricos, estilo de vida, dieta y factores genéticos (Narod 2006); pero se destacan los antecedentes familiares como uno de los factores más importantes. Se ha estimado que la predisposición genética al cáncer mamario es 
responsable del 5-10\% de todos los casos de esta patología (Balmain et al. 2003). La mayoría de casos familiares de neoplasia de mama y de ovario están asociados con mutaciones en los genes BRCA1 (Breast Cancer 1) y BRCA2 (Breast Cancer 2), aunque hay otros genes relacionados con este tipo de cáncer que confieren un riesgo mucho menor.

En Costa Rica, no hay información referente a los factores de riesgo determinantes de CM. Por tanto, el objetivo general de este estudio fue obtener información epidemiológi$\mathrm{ca}$, de factores de riesgo y genética molecular del cáncer de mama hereditario en Costa Rica para identificar los potenciales modificadores de riesgo.

\section{MATERIAL Y MÉTODOS}

Del 2007 al 2009, se seleccionaron 116 mujeres diagnosticadas con CM y con antecedentes familiares de este cáncer en Costa Rica. El reclutamiento de participantes fue realizado por selección directa, referidos por médicos ginecólogos oncólogos, por la Fundación Nacional de la Solidaridad Contra el Cáncer de Mama (FUNDESO), la Fundación Esperanza y otras organizaciones sociales.

Para su selección, cada una de estas familias tenía que cumplir con los siguientes criterios:

1. Al menos tres individuos afectados con la enfermedad a cualquier edad en primer o segundo grado en la misma línea parental.

2. Dos casos de cáncer de mama en primer grado en la misma línea parental, ambos diagnosticados antes de los 50 años.

3. Cada una de las participantes debía tener un diagnóstico de CM confirmado por el Registro Nacional de Tumores y ser mayor de 18 años.

Todas las participantes firmaron un consentimiento informado, debidamente aprobado por el Comité Ético Científico de la Universidad de Ciencias Médicas. Además, se les aplicó un cuestionario de manera directa del cual se obtuvo información sobre factores gineco-obstétricos (edad de la menarca, anticonceptivos orales, embarazos, edad del primer embarazo y lactancia), estilo de vida (ingesta de alcohol, tabaquismo, índice de masa corporal y actividad física) y neoplásicos familiares (familiares de primer y segundo grado con $\mathrm{CM}$ ).

A cada participante, se le tomó una muestra de sangre de aproximadamente $10 \mathrm{~mL}$. Para la extracción de ADN, se utilizó el kit Puregene DNA extraction. Se aplicó el análisis de proteína truncada (PTT) para tamizar el exón 11 en $B R C A 1$ y los exones 10 y 11 de $B R C A 2$, que cubre aproximadamente el $58 \%$ y $50 \%$ de la región codificante de los genes BRCA1 y BRCA2, respectivamente. Todas las muestras fueron analizadas para la duplicación exón 13 6-kb en BRCA1 (Kupperstein 2006), además se analizaron con el método "rapid multiplex" (Kupperstein 2000) las mutaciones 185delAG, 5382insC y ins6kb, en BRCA1 y, 6174delT y 5531delT en BRCA2; dado que la base de datos del Breast Cancer Information Core (BIC) indica que son las mutaciones con el mayor número de registros. La mutación 6174 delT se identificó mediante procedimientos estándar y las restantes se identificaron por PTT. Las variantes que fueron identificadas como positivas para esta prueba, se confirmaron por secuenciación directa del ADN. Todo lo anterior se llevó a cabo en el Women's College Research Institute, Toronto, Canadá.

Los resultados fueron analizados mediante el programa Statistical Package for Social Sciences (SPSS v17.0). Se realizó un análisis de frecuencias, porcentajes y promedios.

\section{RESULTADOS}

Características de la muestra: Las participantes en este proyecto tenían una edad promedio de 53.0 años $(\mathrm{DE}=11.4, \mathrm{n}=116)$. Un $57.4 \%$ y un $46.6 \%$ de los casos tenían como provincia de residencia y nacimiento respectivamente, San José. Esto se debe a un sesgo por la ubicación de los médicos e instituciones referentes. El nivel de escolaridad mayoritario fue el universitario con un $41.7 \%$, seguido del nivel secundario con un $25.2 \%$. 
Con respecto a los factores relacionados con el estilo de vida de las pacientes, se observó que el $72.2 \%(\mathrm{n}=115)$ de las pacientes dijo nunca haber ingerido licor y un $75.7 \%(n=115)$ dijo no tener antecedentes de tabaquismo.

La mayoría de las participantes (68.5\%) realizó algún tipo de actividad física menos de tres veces por semana $(n=108)$. Con respecto al índice de masa corporal, un $43.0 \%$ poseyó un índice normal mientras que un $36.5 \%$ se encontró en la categoría de sobrepeso $(n=91)$, según el criterio establecido por la Organización Mundial de la Salud.

Antecedentes ginecobstétricos: Se mostró una edad de menarca promedio de 12.60 años $(\mathrm{DE}=1.697, \mathrm{n}=114)$, con un mínimo de nueve años y un máximo de 18 años. Un $62.8 \%$ confirmó haber usado anticonceptivos orales, mientras que un $37.2 \%$ dijo no haberlos ingerido $(n=113)$. El número de embarazos promedio para esta población fue de $3.05(\mathrm{DE}=2.153$, $\mathrm{n}=114)$ y hubo doce 12 mujeres nulíparas. El número de partos promedio fue de 2.71 $(\mathrm{DE}=1.847, \mathrm{n}=114)$ y el promedio de número de abortos de $0.34(\mathrm{DE}=0.636, \mathrm{n}=114)$. La edad del primer embarazo mostró un promedio de $22.98(\mathrm{DE}=4.585, \mathrm{n}=101)$ y el mayor porcentaje se encontró en el grupo de edad de 16-20 años con un $36.6 \%$. Con respecto a lactancia, un $26.6 \%$ de las mujeres dijeron haber amamantado a sus hijos un promedio de uno a cinco meses, mientras que un $9.6 \%$ no tuvo periodo de lactancia $(n=94)$. La edad de la menopausia promedio fue de 46.01 años (D.E=7.852, n=80) con un ámbito entre 28-58 años.

Historia médica del cáncer: Con respecto al método de diagnóstico del cáncer, el principal fue el autoexamen mamario (65.2\%, n=112), seguido de la mamografía (20.5\%, $\mathrm{n}=112)$. La mayoría (39.5\%) fueron diagnosticadas en un hospital $(\mathrm{n}=114)$ y la edad del diagnóstico osciló entre 27-75 años, con un promedio de 46.65 años $(\mathrm{DE}=10.236$, $\mathrm{n}=114)$. El 29.8\% de los casos fue diagnosticado antes de los 40 años y un $71.9 \%$ antes de $\operatorname{los} 50$ años.
Diagnóstico molecular: Se analizaron 116 pacientes con $\mathrm{CM}$ para las mutaciones 185delAG, 5382insC e ins6kb, en el gen BRCA1 y, 6174delT y 5531delT en el gen BRCA2. En dos casos no se obtuvieron datos moleculares.

Se encontraron seis pacientes (5.2\%) con una mutación identificada: cinco presentaron una mutación en el gen BRCA2 y sólo un caso presentó una mutación en el gen BRCA1 (Cuadro 1). Con respecto a las mutaciones en el gen BRCA2, tres de los casos presentaron la mutación 5531delTT (dos de ellos estuvieron relacionados familiarmente) y dos casos, también emparentados, presentaron la mutación 6174delT. La única mutación encontrada en el gen BRCA1 correspondió a C3522T. Las mujeres portadoras de una mutación en BRCA tuvieron en promedio una edad de diagnóstico menor.

Antecedentes familiares: Con el propósito de analizar la asociación entre el cáncer de mama y el historial familiar de esta enfermedad u otros tipos de cáncer, se analizaron los antecedentes patológicos de las pacientes. Dentro de la muestra seleccionada, 71 (61\%) casos presentaron parientes de primer grado diagnosticados con cáncer de mama y $65(56 \%)$ casos tuvieron antecedentes de segundo grado.

\section{CUADRO 1}

Mutaciones identificadas en los genes BRCA1 y BRCA2 en pacientes costarricenses diagnosticadas con cáncer de mama

TABLE 1

BRCA1 and BRCA2 Mutations Identified in Costa Rican Breast Cancer Patients

\begin{tabular}{cccc} 
Paciente & Gen & Exón & Mutación \\
CM24 & BRCA1 & 11 & C3522T \\
CM10-2 & BRCA2 & 11 & 5531delTT \\
CM10-3 & BRCA2 & 11 & 5531delTT \\
CM88 & BRCA2 & 11 & 5531 delTT \\
CM20-1 & BRCA2 & 11 & 6174 delT \\
CM20-2 & BRCA2 & 11 & $6174 d e l T$ \\
\hline
\end{tabular}




\section{DISCUSIÓN}

En un grupo de 116 pacientes con cáncer de mama, se analizó la prevalencia de los factores de riesgo y de las mutaciones en los genes BRCA1 y BRCA2.

Con respecto a la actividad física, Thune et al. (1997) encontraron que ésta protege contra el cáncer de mama, particularmente entre mujeres premenopáusicas y posmenopáusicas dado que podría afectar los niveles hormonales. Aunque en el presente estudio no se encontró relación alguna, es importante hacer hincapié en el efecto preventivo de la actividad física.

No parece que el ser portadora de una mutación influya en la edad de la menarca. Esto concuerda con lo reportado por Kotsopoulos et al. (2005) en un estudio con aproximadamente 4000 pacientes. Sin embargo, encontraron en las mujeres con una mutación en el gen BRCA1, que la edad de la menarca estaba inversamente relacionada con el riesgo de la patología. En nuestra muestra los datos no indican esta asociación debido a que solo hay una paciente portadora de una mutación en BRCA1.

En el 2007, a nivel nacional, se obtuvo una tasa global de fecundidad de 1.96; de estos nacimientos el 56\% correspondieron a mujeres entre los 20-29 años, y el 20.2\% a mujeres menores de 20 años (Ministerio de Salud 2008). En el caso de pacientes diagnosticados con cáncer mamario, se obtuvo un promedio mayor para las variables número de partos $(2.71 \pm 1.847)$ y edad del primer embarazo (22.98 \pm 4.585$)$ que el de la población costarricense en general. El mayor porcentaje de nacimientos en esta muestra se da en el grupo de edad de 16 a 20 años, lo cual concuerda con un estudio previo (Kotsopoulos et al. 2007), que indica que un embarazo temprano no proporciona protección contra el cáncer de mama en pacientes portadoras de una mutación en BRCA1/2 ni en la población en general. El porcentaje obtenido $(2.5 \%)$ de mujeres menopáusicas en el grupo de edad antes de los 35 años, podrían deberse a una menopausia inducida por el diagnóstico temprano de estas pacientes.
Este estudio determina que el autoexamen de mama es el principal método de diagnóstico y se debe hacer notar la relevancia que tienen las campañas educativas a la población y la importancia de la concientización del valor de un diagnóstico temprano por parte de las entidades gubernamentales.

Sobre la edad promedio de diagnóstico, ésta concuerda con la edad pre-menopáusica de las mujeres, sin embargo, no concordó con los datos para la población costarricense donde la mayor incidencia se da en el grupo de 50 a 54 años con una tasa de 117.37 (tasa por 100 000 mujeres) (Organización Panamericana de la Salud 2008).

Las pacientes portadoras de una mutación, tuvieron un diagnóstico aproximadamente diez años antes que las no portadoras, lo cual concuerda con estudios previos donde se observa que mujeres diagnosticadas antes de los 35 años presentaron alguna mutación en los genes BRCA1/2 (Peto et al. 1999). Esto por cuanto, el cáncer de mama hereditario está asociado con un diagnóstico temprano y una historia familiar de la enfermedad, en concordancia con una herencia mendeliana dominante.

Se identificaron mutaciones en un 5.2\% de las mujeres seleccionadas por antecedentes familiares de patología mamaria. En un estudio en familias seleccionadas con cáncer de mama en Chile se reporta un 10.4\% (Gallardo et al. 2006) y en estudios de prevalencia con pacientes no seleccionadas se indica entre el $2.3 \%$ y el 2.5\% (Gomes et al. 2007, Comacho et al. 2008) de mutaciones en los genes BRCA1/2. Las frecuencias de mutaciones en estos genes son bajas pero se asemejan a la incidencia encontrada en otros países. Hay que tomar en cuenta que el análisis mutacional se restringió a las mutaciones más comunes.

Las mutaciones en el gen BRCA2 se presentaron en mayor proporción que en el gen BRCA1, lo que indicaría que la prevalencia de mutaciones en BRCA2 es mayor en esta población. Sin embargo, debe tomarse en cuenta que el tamaño de la muestra es pequeño para obtener una diferencia estadísticamente significativa. El significado clínico de las mutaciones 
encontradas es un cambio en el encuadre de lectura, a excepción de la mutación C3522T la cual es de pérdida de sentido.

Serán necesarios futuros estudios con una muestra más grande y con el uso de un grupo control apareado por edad y región geográfica para poder determinar las características propias de los tumores relacionados con las mujeres costarricenses portadoras de mutaciones en los genes BRCA1/2. También, debe insistirse en hacer un reporte histológico completo donde se pueda obtener información adicional de la histología, así como un reporte hormonal completo que se complemente con el reporte histológico para obtener información integral de las características clínico-patológicas.

La capacidad para identificar un fenotipo histológico específico de carcinoma mamario en portadores de mutaciones en los genes BRCA1 y BRCA2, y la información patológica específica, podrían ser una guía para protocolos de pruebas genéticas y utilizarse como un criterio adicional para priorizar familias para el estudio genético y una manera de personalizar estrategias de manejo para estas pacientes.

El poseer antecedentes familiares de cáncer de mama, tampoco muestra una relación estadísticamente significativa con ser portador de una mutación en BRCA1/2. Dada la gran proporción de familias con alto riesgo en las cuales no se identificó ninguna mutación, se sugiere que otros genes, no contemplados en este estudio, pueden estar afectando la incidencia de la enfermedad. Se debe tomar en cuenta que los factores ambientales, como el estilo de vida y la dieta, pueden ser determinantes de poseer un riesgo familiar, pues están directamente implicados no solo en la expresión del cáncer, sino también en el tipo de tejido afectado y el grado de severidad del cáncer (Gallardo et al. 2006); por lo que existe la necesidad de criterios adicionales para una mejor identificación de las mujeres que porten una mutación en estos genes (Armes et al. 1998, Lalloo \& Gareth 1999).

El mejor entendimiento de los factores genéticos de riesgo y su interacción con el ambiente va a permitir la predicción y prevención de enfermedades tanto a nivel individual como poblacional (Pharoah et al. 2002). Costa Rica posee una población joven y la mayoría de los diagnósticos se dan antes de los 50 años de edad, con lo que se espera ver una proporción relativamente alta de casos hereditarios; establecer el porcentaje hereditario de cáncer de mama en casos no seleccionados es de interés para futuras investigaciones (Gutiérrez-Espeleta et al. 2011).

Estos resultados y el aumento en la incidencia del cáncer de mama en los últimos años en Costa Rica deben promover métodos de detección más eficientes, que permitan identificar la patología de una forma anticipada con el objetivo de facilitar la toma de decisiones en cuanto a campañas de prevención y tratamiento médico.

\section{AGRADECIMIENTOS}

A Melissa Enmore por el soporte técnico. A Alvaro Apestegui por su colaboración en la toma de las muestras de sangre. A la Fundación Nacional de Solidaridad Contra el Cáncer de Mama (FUNDESO) y Fundación Esperanza, Movimiento Elige Vivir por las referencias de las pacientes.

\section{RESUMEN}

El cáncer de mama ocupa el primer lugar en incidencia y mortalidad entre las mujeres costarricenses, para el año 2011, se tiene una proyección de 1071 casos nuevos. Mediante selección directa se reclutaron 116 pacientes diagnosticadas con cáncer de mama y con antecedentes familiares de esta enfermedad. Se analizó la prevalencia de los factores de riesgo y de las mutaciones en los genes BRCA1 y BRCA2. Se encontró que la edad promedio de las mujeres portadoras de una mutación es menor que en mujeres no portadoras. Además, se identificaron seis pacientes $(5.2 \%)$ con una mutación en el gen BRCA2 y sólo una presentó una mutación en el gen BRCA1. Estos resultados y el aumento en la incidencia del cáncer de mama en los últimos años en Costa Rica deben promover un aumento en medidas de prevención y el establecimiento de métodos de detección más eficientes, que permitan realizar un diagnóstico temprano de la enfermedad.

Palabras clave: cáncer de mama, hereditario, factores de riesgo, Costa Rica. 


\section{REFERENCIAS}

Armes, J., M. Egan, M. Southey, G. Dite, M. McCredie, G. Giles, J. Hopper \& J. Venter. 1998. The histologic phenotypes of breast carcinoma occurring before age 40 years in women with and without BRCA1 or BRCA2 germline mutations a population-based study. Cancer 83: 2335-2345.

AICR. 2008. World Cancer Research Fund/American Institute for Cancer Research. Food, nutrition, physical activity, and the prevention of cancer: A Global perspective. American Institute for Cancer Research, Washington D.C., EEUU.

Balmain, A., J. Gray \& B. Ponder. 2003. The genetics and genomics of cancer. Nat. Genet. 33: 238-244.

Comacho, R., A. Esperon, R. Ropero, M. Rubio, R. Rodríguez, R. Ortiz, J. Lence, M. de los Rios, D. Carnesolta, M. del Olivera, S. Vansam, R. Royer, M. Akbari, T. Donenberg \& S. Narod. 2008. Prevalence of BRCA1 and BRCA2 mutations in breast cancer patients from Cuba. Fam. Cancer 7: 275-279.

Gallardo, M., A. Silva, L. Rubio, C. Alvarez, C. Torrealba, M. Salinas, T. Tapia, P. Faundez, L. Palma, M. Riccio, H. Paredes, M. Rodriguez, A. Cruz, C. Rousseau, M. King, M. Camus, M. Alvarez \& P. Carvallo. 2006. Incidence of $B R C A 1$ and $B R C A 2$ mutations in 54 Chilean families with breast/ovarian cancer, genotype-phenotype correlations. Breast Cancer Res. Treat. 95: 81-7.

Gomes, M., M.M. Costa, R. Borojevic, A.N.A. Monteiro, R. Vieira, S. Koifman, R. Koifman, S. Li, R. Royer, S. Zhang \& S.A. Narod 2007. Prevalence of BRCA1 and $B R C A 2$ mutations in breast cancer patients from Brazil. Breast Cancer Res. Treat. 103: 349-353.

Gutiérrez-Espeleta, G.A., M. Llacuachaqui, L. GarcíaJiménez, M. Aguilar Herrera, K. Loáiciga Vega, A. Ortiz, R. Royer, S. Li \& S.A. Narod. 2011. BRCA1 and BRCA2 Mutations among Familial Breast Cancer Patients from Costa Rica. Clin. Genet. Sept 5 (Pub. ahead of print).

Kotsopoulos, J., J. Lubinski, H. Lynch, S. Neuhausen, P. Ghadirian, C. Isaacs, B. Weber, C. Kim-Sing, W. Foulkes, R. Gershoni-Baruch, P. Ainsworth, E. Friedman, M. Daly, J. Garber, B. Karlan, O. Olopade, N. Tung, H. Saal, A. Eisen, M. Osborne, H. Olsson, D. Gilchrist, P. Sun 1 \& S. Narod. 2005. Age at menarche and the risk of breast cancer in BRCA1 and BRCA2 mutation carriers. Canc. Causes Contr. 16: 667-674.
Kotsopoulos, J., J. Lubinski, H.T. Lynch, J. Klijn, P. Ghadirian, S.L. Neuhausen, C. Kim-Sing, W.D. Foulkes, P. Moller, C. Isaacs, S. Domchek, S. Randall, K. Offit, N. Tung, P. Ainsworth, R. Gershoni-Baruch, A. Eisen, M. Daly, B. Karlan, H.M. Saal, F. Couch, B. Pasini, T. Wagner, E. Friedman, G. Rennert, C. Eng, J. Weitzel, P. Sun \& S. Narod. 2007. Age at first birth and the risk of breast cancer in BRCA1 and BRCA2 mutation carriers. Breast Cancer Res. Treat. 105: 221-228.

Kupperstein, G., W.P. Foulkes, P. Ghandirian, J. Hakimi \& S.A. Narod. 2000. A rapid fluorescent multiplex PCR analysis (FMPA) for founder mutations in the BRCA1 and BRCA2 genes. Clin. Genet. 57: 213-220.

Kuperstein, G., E. Jack \& S.A. Narod. 2006. A fluorescent multiplex-DGGE screening test for mutations in the BRCA1 gene. Genet. Test. 10: 1-7.

Lalloo, F. \& D. Gareth. 1999. The pathology of familial breast cancer Clinical and genetic counselling implications of breast cancer pathology. Breast Cancer Res. Treat. 1: 5-9.

McPhearson, K., C.M. Steel \& J.M. Dixon. 2000. Breast cancer-epidemiology, risk factors and genetics. Brit. Med. J. 321: 624- 628.

Ministerio de Salud. 2007. Plan Nacional para el Control del Cáncer 2007-2016. San José, Costa Rica.

Ministerio de Salud. 2008. Memoria Institucional. San José, Costa Rica.

Narod, S.A. 2006. Modifiers of risk of hereditary breast cancer. Oncogene 25: 5832-5836.

Organización Panamericana de la Salud. 2008. Indicadores básicos de Costa Rica.

Ortiz, A.E. 2007. Supervivencia de las mujeres con cáncer de mama según desarrollo humano, Costa Rica 20002003: un insumo para la gerencia de la salud. Tesis de Maestría, Universidad de Costa Rica, San José, Costa Rica.

Peto, J., N. Collins, R. Barfoot, S. Seal, W. Warren, N. Rahman, D.F. Easton, C. Evans, J. Deacon \& M.R. Stratton. 1999. Prevalence of BRCA1 and BRCA2 Gene Mutations in patients with early-onset breast cancer. J. Natl. Cancer Inst. 91: 943-949.

Pharoah, P., A. Antoniou, M. Bobrow, R.L. Zimmern, D.F. Easton \& B.A.J. Ponder. 2002. Polygenic susceptibility to breast cancer and implications for prevention. Nat. Gen. 31: 33-36.

Thune, I., T. Brenn, E. Lund \& M. Gaard. 1997. Physical activity and the risk of breast cancer. N. Engl. J. Med. 336: 1269-1275. 